PROCEEDINGS OF THE

AMERICAN MATHEMATICAL SOCIETY

Volume 126, Number 2, February 1998, Pages 421-423

S 0002-9939(98)04527-4

\title{
A NOTE ON A JACOBIAN IDENTITY
}

\author{
JOHN A. EWELL
}

(Communicated by Hal L. Smith)

\begin{abstract}
An identity involving eight-fold infinite products, first derived by Jacobi in his theory of theta functions, is the subject of this note. Three similar identities, including one that implies Jacobi's identity, are presented.
\end{abstract}

\section{INTRODUCTION}

In this note our point of departure is the identity of the following theorem.

Theorem 1.1 (Jacobi). For each complex number $x$ such that $|x|<1$,

$$
\left\{\prod_{1}^{\infty}\left(1-x^{2 n-1}\right)\right\}^{8}+16 x\left\{\prod_{1}^{\infty}\left(1+x^{2 n}\right)\right\}^{8}=\left\{\prod_{1}^{\infty}\left(1+x^{2 n-1}\right)\right\}^{8} .
$$

According to Whittaker and Watson [3, p. 470], Jacobi was deeply impressed by his discovery of this result. We here propose to show that in the proper setting identity (1.1) is just one of four similar identities, all of which are equally striking. As a matter of fact, we show that one of the three remaining identities implies Jacobi's identity.

\section{Proofs}

The proper setting alluded to in the foregoing paragraph is occupied by the following sextuple-product identity:

$$
\begin{gathered}
\prod_{1}^{\infty}\left(1-x^{2 n}\right)^{2}\left(1+a b x^{2 n-1}\right)\left(1+a^{-1} b^{-1} x^{2 n-1}\right)\left(1+a b^{-1} x^{2 n-1}\right)\left(1+a^{-1} b x^{2 n-1}\right) \\
=\sum_{-\infty}^{\infty} x^{2 m^{2}} a^{2 m} \sum_{-\infty}^{\infty} x^{2 n^{2}} b^{2 n}+x \sum_{-\infty}^{\infty} x^{2 m(m+1)} a^{2 m+1} \sum_{-\infty}^{\infty} x^{2 n(n+1)} b^{2 n+1},
\end{gathered}
$$

which is valid for each triple of complex numbers $a, b, x$ such that $a \neq 0, b \neq 0$ and $|x|<1$. In [1] the author shows that identity (2.1) is an easy and straightforward consequence of the celebrated Gauss-Jacobi triple-product identity:

$$
\prod_{1}^{\infty}\left(1-x^{2 n}\right)\left(1+t x^{2 n-1}\right)\left(1+t^{-1} x^{2 n-1}\right)=\sum_{-\infty}^{\infty} x^{n^{2}} t^{n}
$$

Received by the editors July 25, 1996.

1991 Mathematics Subject Classification. Primary 33D10; Secondary 05A19.

Key words and phrases. Identities of Jacobi, theta functions.

(C)1998 American Mathematical Society 
which is valid for each pair of complex numbers $t, x$ such that $t \neq 0$ and $|x|<1$. There are now many elementary proofs of identity (2.2). E.g., see [2, pp. 282-283].

Theorem 2.1. For each complex number $x$ such that $|x|<1$,

$$
\prod_{1}^{\infty}\left(1-x^{4 n-2}\right)^{2}\left(1+x^{2 n-1}\right)^{4}=\prod_{1}^{\infty}\left(1+x^{4 n-2}\right)^{4}+4 x \prod_{1}^{\infty}\left(1+x^{4 n}\right)^{4} .
$$

Proof. By appeal to (2.2) we express each series on the right side of (2.1) as an infinite product

$$
\begin{aligned}
\prod_{1}^{\infty}(1- & \left.x^{2 n}\right)^{2}\left(1+a b x^{2 n-1}\right)\left(1+a^{-1} b^{-1} x^{2 n-1}\right)\left(1+a b^{-1} x^{2 n-1}\right)\left(1+a^{-1} b x^{2 n-1}\right) \\
= & \prod_{1}^{\infty}\left(1-x^{4 n}\right)^{2}\left(1+a^{2} x^{4 n-2}\right)\left(1+a^{-2} x^{4 n-2}\right)\left(1+b^{2} x^{4 n-2}\right)\left(1+b^{-2} x^{4 n-2}\right) \\
& +\left(a+a^{-1}\right)\left(b+b^{-1}\right) x \\
& \times \prod_{1}^{\infty}\left(1-x^{4 n}\right)^{2}\left(1+a^{2} x^{4 n}\right)\left(1+a^{-2} x^{4 n}\right)\left(1+b^{2} x^{4 n}\right)\left(1+b^{-2} x^{4 n}\right) .
\end{aligned}
$$

In (2.4) we let $a=b$, and cancel $\prod\left(1-x^{4 n}\right)^{2}$ to get

$$
\begin{aligned}
\prod_{1}^{\infty}(1- & \left.x^{4 n-2}\right)^{2}\left(1+x^{2 n-1}\right)^{2}\left(1+a^{2} x^{2 n-1}\right)\left(1+a^{-2} x^{2 n-1}\right) \\
= & \prod_{1}^{\infty}\left(1+a^{2} x^{4 n-2}\right)^{2}\left(1+a^{-2} x^{4 n-2}\right)^{2} \\
& +\left(a+a^{-1}\right)^{2} x \prod_{1}^{\infty}\left(1+a^{2} x^{4 n}\right)^{2}\left(1+a^{-2} x^{4 n}\right)^{2} .
\end{aligned}
$$

Now, in the foregoing identity we let $a=1$ to prove our theorem.

In identity (2.3) let $x \rightarrow-x$ to get

$$
\prod_{1}^{\infty}\left(1-x^{4 n-2}\right)^{2}\left(1-x^{2 n-1}\right)^{4}=\prod_{1}^{\infty}\left(1+x^{4 n-2}\right)^{4}-4 x \prod_{1}^{\infty}\left(1+x^{4 n}\right)^{4} .
$$

Multiply the foregoing identity and (2.3) to get

$$
\prod_{1}^{\infty}\left(1-x^{4 n-2}\right)^{8}=\prod_{1}^{\infty}\left(1+x^{4 n-2}\right)^{8}-16 x^{2} \prod_{1}^{\infty}\left(1+x^{4 n}\right)^{8} .
$$

Now, in this identity let $x \rightarrow x^{1 / 2}$ to obtain Jacobi's identity (1.1).

Theorem 2.2. For each complex number $x$ such that $|x|<1$,

$$
\begin{aligned}
\prod_{1}^{\infty}\left(1+x^{2 n-1}\right)\left(1+x^{6 n-3}\right)\left(1-x^{2 n-1}\right)^{4} \\
=\prod_{1}^{\infty}\left\{\frac{1-x^{12 n-6}}{1-x^{4 n-2}}\right\}^{2}-3 x \prod_{1}^{\infty}\left\{\frac{1-x^{12 n}}{1-x^{4 n}}\right\}^{2}
\end{aligned}
$$


and

$$
\begin{aligned}
\prod_{1}^{\infty}\left(1-x^{2 n-1}\right)^{5}\left(1-x^{6 n-3}\right) \\
\quad=\prod_{1}^{\infty}\left\{\frac{1-x^{6 n-3}}{1-x^{2 n-1}}\right\}^{4}-9 x \prod_{1}^{\infty}\left\{\frac{1-x^{6 n}}{1-x^{2 n}}\right\}^{4} .
\end{aligned}
$$

Proof. To prove (2.5) we return to identity (2.4) where we first let $a \rightarrow i a^{2}$ and $b \rightarrow i a$ to get

$$
\begin{aligned}
\prod_{1}^{\infty}(1- & \left.x^{2 n}\right)^{2}\left(1-a^{3} x^{2 n-1}\right)\left(1-a^{-3} x^{2 n-1}\right)\left(1+a x^{2 n-1}\right)\left(1+a^{-1} x^{2 n-1}\right) \\
= & \prod_{1}^{\infty}\left(1-x^{4 n}\right)^{2}\left(1-a^{4} x^{4 n-2}\right)\left(1-a^{-4} x^{4 n-2}\right)\left(1-a^{2} x^{4 n-2}\right)\left(1-a^{-2} x^{4 n-2}\right) \\
& -\left(a-a^{-1}\right)^{2}\left(a+a^{-1}\right) x \\
& \times \prod_{1}^{\infty}\left(1-x^{4 n}\right)^{2}\left(1-a^{4} x^{4 n}\right)\left(1-a^{-4} x^{4 n}\right)\left(1-a^{2} x^{4 n}\right)\left(1-a^{-2} x^{4 n}\right)
\end{aligned}
$$

Next, recall that for $\rho:=(-1+i \sqrt{3}) / 2$, we have:

$$
\rho^{3}=1 \quad \text { and } \quad \rho^{2}+\rho+1=0 .
$$

In the foregoing identity we then let $a \rightarrow \rho$ to get

$$
\begin{aligned}
\prod_{1}^{\infty}\left(1-x^{2 n}\right)^{2}\left(1-x^{2 n-1}\right)^{2} \frac{1+x^{6 n-3}}{1+x^{2 n-1}} & \\
& =\prod_{1}^{\infty}\left(1-x^{4 n}\right)^{2}\left\{\frac{1-x^{12 n-6}}{1-x^{4 n-2}}\right\}^{2}-3 x \prod_{1}^{\infty}\left(1-x^{4 n}\right)^{2}\left\{\frac{1-x^{12 n}}{1-x^{4 n}}\right\}^{2} .
\end{aligned}
$$

Cancelling $\prod\left(1-x^{4 n}\right)^{2}$ and simplifying, we obtain identity (2.5).

In identity (2.5) let $x \rightarrow-x$, and multiply the resulting identity and (2.5) to get

$$
\begin{aligned}
\prod_{1}^{\infty}\left(1-x^{4 n-2}\right)^{5}\left(1-x^{12 n-6}\right) & \\
& =\prod_{1}^{\infty}\left\{\frac{1-x^{12 n-6}}{1-x^{4 n-2}}\right\}^{4}-9 x^{2} \prod_{1}^{\infty}\left\{\frac{1-x^{12 n}}{1-x^{4 n}}\right\}^{4} .
\end{aligned}
$$

Finally, in the foregoing identity let $x \rightarrow x^{1 / 2}$ to obtain identity (2.6).

\section{REFERENCES}

1. J. A. Ewell, Arithmetical consequences of a sextuple product identity, Rocky Mountain J. Math., v. 25, 1995. MR 97e:11129

2. G. H. Hardy and E. M. Wright, An introduction to the theory of numbers, 4th ed., Clarenden Press, Oxford, 1960. MR 81i:10002 (5th edition review)

3. E. T. Whittaker and G. N. Watson, A course of modern analysis, Cambridge University Press, New York, 1973.

Department of Mathematical Sciences, Northern Illinois University, DeKalb, IlliNOIS 60115 